\title{
O texto semiótico da cibercultura: uma análise do processo modelizante da autoria no website Overmundo
}

Beatriz Cintra Martins

\section{Resumo:}

O artigo traz uma análise da publicação eletrônica Overmundo, tendo como referencial teórico a Semiótica da Cultura. A abordagem é focada especificamente na produção discursiva co-autorada, ou autoria colaborativa, existente neste website, através da exploração das linguagens que interagem para tecer a sua interface. Propõe-se, deste modo, uma interpretação do processo modelizante da autoria nesse ambiente como uma ação coletiva dialógica em um espaço público virtual.

\section{Palavras Chave:}

Semiótica da Cultura; cibercultura; autoria colaborativa; Overmundo

\begin{abstract}
:
The article analyzes the electronic publication Overmundo, using the Semiotics of Culture as theoretical reference. The approach is specifically focused on the discursive co-authored production, or collaborative authoring, available on that site, exploring languages that interact to weave its interface. Thus, the article attempts an interpretation of the authoring modelizing process in that environment as a dialogical collective action in a public virtual space.
\end{abstract}

\section{Keywords:}

Semiotics of Culture; cyberculture; collaborative authoring; Overmundo

\section{Introdução}

Cada sociedade, em diferentes épocas históricas, constrói complexas relações entre múltiplas linguagens e códigos, que podem ser compreendidas, de acordo com o que propõe a Semiótica da Cultura, como textos culturais. Estas relações são compostas de processos dinâmicos, nada estáticos, que vão incessantemente redefinindo códigos e significações, e assim produzindo uma diversidade de linguagens e sistemas culturais.

Seguindo este referencial teórico iremos analisar a cibercultura como um texto da cultura contemporânea. Por cibercultura entendemos a relação entre as novas tecnologias de comunicação e a vida social contemporânea, de acordo com a definição de Lemos (2002). Circunscrita inicialmente a alguns ambientes de pesquisa governamental e universitária, com grande influência da chamada contracultura, especialmente da cultura hacker, a cibercultura chegou ao centro da cultura ocidental contemporânea, na velocidade das redes de comunicação. Trouxe consigo um conjunto de conteúdo simbólico que entrou em semiose com outros sistemas culturais, produzindo novos sentidos.

Esse encontro entre diferentes sistemas culturais, que resultou na criação de uma nova tecnologia de comunicação, pode ser visto, como propõe a Semiótica da Cultura, como um momento de explosão: um salto de um passado para um futuro; um choque entre diferentes códigos e linguagens, uma confluência de traços diversos; uma junção que não representa uma soma nem uma subtração, ou uma imposição de um sobre o outro, mas sim um resultado imprevisível e não planejado que produz um novo que é fruto da reunião de 
diferentes traços culturais dos sistemas de origem.

Como uma nova tecnologia, representa também a complexificação do texto da cultura, ao expandir os sistemas sígnicos e criar novos processos modelizantes, incrementando a diversidade de linguagens e códigos culturais. Neste sentido é que o seu advento pode ser mais bem compreendido com a imagem de uma explosão fértil e expansiva, que entra em relação com todo o repertório da memória cultural para daí gerar o novo, muito mais do que um evento de caráter impactante, produtor de efeitos dentro de uma lógica causal. (Cf. MACHADO, 2006)

A fim de analisar a semiótica da cibercultura iremos focar nosso olhar sobre um traço específico desse sistema que tem sido objeto de estudo de um sem número de pesquisas sobre as redes de comunicação, a saber, a prática colaborativa. A cooperação, cabe esclarecer, faz parte da própria semiose e, sem dúvida alguma, pode ser observada nos mais diversos processos de construção de sentido nas variadas formas de interação humana nos mais diferentes sistemas de linguagem. Neste trabalho, buscaremos explorar quais são suas peculiaridades no contexto da cibercultura, mais especificamente na chamada edição colaborativa ou escrita coletiva (1), característica de grande parte das publicações eletrônicas existentes na Internet, como blogs, sistemas wiki etc. Queremos abordar este fenômeno como um processo autoral em rede, que se dá na forma da interação entre diferentes falantes em um diálogo que tem lugar no ambiente cibernético do espaço-informação (2).

Para construir nossa análise, nosso objeto de estudo será o website Overmundo (3), que tem a edição colaborativa como um de seus traços marcantes. Num primeiro momento, iremos aprofundar o quadro teórico da Semiótica da Cultura para, posteriormente, fazer algumas reflexões sobre a web como produtora de semiose. Em seguida, proporemos uma interpretação do processo modelizante da autoria nesse ambiente.

\section{A cultura como texto}

Pensar a cultura como texto é entendê-la como uma tessitura de diversos sistemas culturais, hierarquicamente organizados, cada um deles por sua vez composto de diferentes linguagens. O texto é a composição dinâmica desses diferentes elementos que interagem entre si em um processo auto-organizativo e auto-poiético, compondo um sistema dialógico de caráter codificado. Inconcluso e aberto, internamente contraditório, o texto cumpre duas funções básicas: a transmissão de significados e a geração de novos sentidos.

Para Lotman (1978) linguagem é "todo o sistema de comunicação que utiliza signos ordenados de modo particular”. (LOTMAN, 1978, p. 35) Entende-se a partir desta conceituação que os signos na linguagem estão em relação em uma estrutura hierárquica que possibilita uma diversidade de combinatórias, e não outras. Por estar sujeita a regras de combinação dada pelo seu código, a linguagem deve ser diferenciada de sistemas que não servem de meio de comunicação; de sistemas que, mesmo servindo de meio de comunicação, não usam signos; e, por último, de sistemas que, mesmo servindo de meio de comunicação e utilizando signos, são pouco ou nada ordenados.

Dentro do conceito de linguagem, Lotman distingue três campos distintos: as línguas naturais (os idiomas); as línguas artificiais (as línguas da ciência; a matemática; os sinais de trânsito etc.); as linguagens secundárias, as estruturas de comunicação ou os sistemas culturais que se sobrepõe à língua natural (os mitos, a arte, os rituais etc.). É preciso ressaltar aqui que para a Semiótica da Cultura nenhuma linguagem tem prevalência na cultura, ou dito de outro modo, todas as linguagens tem valor cultural equivalente. Neste sentido, as linguagens secundárias não são subordinadas ou segundas em relação à língua natural. Apenas esta última serve de referência para a leitura das primeiras, como modelo para a sua descrição. Por outro lado, nenhum sistema cultural pode ser visto como uma unidade isolada, ao contrário, está sempre em relação com os outros sistemas culturais no contexto da semiosfera (4). 
Já as linguagens podem ser compostas de signos discretos e não discretos, ou contínuos. Os signos discretos são aqueles que podem ser decompostos, como é o caso do alfabeto. Os signos contínuos são aqueles que não podem ser percebidos senão em sua totalidade, isto é, se decompostos perdem seu sentido. Este é o caso principalmente do signo visual, como a fotografia ou a pintura. Porém é preciso ter cuidado nessa diferenciação, pois cada linguagem terá sua complexidade que deve ser analisada no dinamismo da interação dos signos, sendo muitas vezes impossível separar seus elementos de forma estanque. Mais produtivo é explorar a interação específica entre os diversos elementos que cada linguagem e cada meio vai possibilitar. O cinema, por exemplo, segundo Lotman, pode ser analisado como a combinação de signos discretos (os planos) e contínuos (a montagem).

No estudo dos sistemas culturais na Semiótica da Cultura, um conceito-chave é o de modelização, que está relacionado ao processo de semiose no qual uma linguagem ressignifica um modelo. A modelização é um instrumento teórico para se compreender a dinâmica dos sistemas culturais, em seu processo de construção de linguagem - como se constituem, como interagem, como se organizam para produzir sentido de forma específica. A modelização é sempre uma recodificação. Podemos observá-la no deslocamento de linguagens entre diferentes sistemas: a angulação da fotografia nos quadrinhos; a narrativa da literatura no cinema etc. Expressa a possibilidade de diálogo entre diferentes sistemas culturais, demonstrando como a cultura é este tecido de relações e de trocas sígnicas entre diferentes sistemas e linguagens. Tem a ver, sobretudo, com a significação que determinado sistema constrói na cultura, como algo que ele traz pela primeira vez, não previsível. Assim, os sistemas são processo modelizante, mediadores entre a informação e o texto cultural. Para Lotman:

Deste modo, cada sistema de comunicação pode realizar uma função modelizante, e inversamente, cada sistema modelizante pode desempenhar um papel de comunicação. Certamente que esta ou aquela função pode ser expressa mais intensamente ou não ser quase sentida nesta ou naquela utilização social concreta. No entanto as duas funções existem potencialmente. (LOTMAN, 1978, p.45)

Outro conceito fundamental nesta teoria é o de estruturalidade, que diz respeito à dinâmica organizadora do sistema: a estrutura que embasa o texto da cultura, entendida como algo flexível e móvel, a fim de permitir o constante diálogo entre os diferentes sistemas de linguagem, suas possibilidades combinatórias, em seu processo auto-organizativo.

No texto da cultura, cada sistema de linguagem tem a sua especificidade, suas características únicas que fazem com que, como afirma Lotman, ele seja capaz de "transmitir informações particulares, não transmissíveis por outros meios" (LOTMAN, 1978, p. 55). Assim, cabe ao pesquisador investigar em cada objeto cultural a sua peculiaridade, a maneira própria com que combina signos e códigos para criar um modelo de mundo. A seguir buscaremos explorar, dentro deste quadro teórico, o processo modelizante da web.

\section{Análise do sistema modelizante da web}

A fim de podermos realizar, posteriormente, uma análise do website Overmundo, nosso objeto de estudo, iremos inicialmente tentar compreender o sistema modelizante da web, isto é, da linguagem WWW (World Wide Web) ou protocolo HTTP (HyperText Transfer Protocol), circunscrevendo nossa reflexão sobre um sistema específico dentre muitos que compõem a rede de comunicação via Internet.

Antes de entrar propriamente nessa análise, cabe registrar a característica mais global do meio digital: sua ancoragem sob o código binário ( 0 e 1$)$, que lhe confere estruturalidade, ou seja, garante a organização primordial de todo o sistema. Neste sentido, afirma-se que a linguagem artificial da matemática modeliza os 
demais sistemas de linguagem presentes nesse ambiente. Também vale deixar pontuado no início desta reflexão, para recuperarmos mais adiante, a presença da eletricidade como participante da semiose no meio digital.

O sistema $w w w$ é composto de linguagens geradas por signos discretos, língua natural (escrita e falada); signos contínuos, imagens (fotos, ilustrações, marcas, ícones); signos discretos e contínuos, vídeos e filmes. Estas linguagens se estruturam, se relacionam ou interagem, através do hipertexto, que organiza o sistema através de elos de conexão semântica que ligam diferentes camadas de texto, entendendo-se como texto todo o conjunto de conteúdo de signos. Assim, por exemplo, em uma unidade desse sistema, alguns signos (palavras ou imagens) possuem links que servem de conexão a outras unidades com signos de mesmo tipo ou diferente. Isto é, em uma página web, através do sistema do hipertexto, uma palavra (signo discreto) ou uma imagem ( signo contínuo) pode conectar a uma outra página que, por sua vez, pode ser composta de diferentes linguagens: texto em língua natural; imagens as mais diversas; gravações sonoras; filmes etc.

O hipertexto pode ser compreendido como o código na medida em que organiza a interação entre os diferentes elementos componentes do sistema. Importante salientar que este código funciona não como um conjunto de regras fixas, mas, sim, como possibilidades combinatórias entre os elementos. Numa primeira observação, constata-se que este código tem como combinatórias os seguintes critérios: não-linearidade; multidirecionalidade; fragmentação; intertextualidade; diversidade; interatividade; transitoriedade e velocidade.

Modelizada em primeira instância pela linguagem artificial, a web realiza modelizações no contexto mais amplo da cultura. Tentaremos apontar alguns desses processos, com a intenção de fazer apenas uma exploração inicial desse tema, bastante complexo para ser abordado em sua amplitude neste breve ensaio.

\subsection{A modelização do espaço virtual}

Um dos processos modelizantes mais marcantes promovidos pela web é aquele relativo à percepção espacial: com a combinação de diferentes linguagens - como a fotografia; o desenho; o cinema; a perspectiva; a programação visual; entre outras - a web modeliza, em um ambiente virtual, diferentes espaços do mundo físico material. Uma das formas mais expressivas desse potencial é a chamada realidade virtual, da qual podemos citar dois exemplos bastante populares: o Google Earth(5) e o Second Life(6). Faremos uma breve análise dos dois, sem a intenção de aprofundar a reflexão, apenas para fins de ilustração.

No primeiro, através das linguagens da fotografia, do desenho e da perspectiva, é modelizado uma espécie de telescópio, através do qual é possível explorar visualmente uma modelização do próprio planeta Terra - mais do que um mapa bidimensional, é o próprio planeta tridimensional (modelizado aqui em perspectiva) que se apresenta para a observação. Pela combinação dessas linguagens, esse instrumento óptico permite aproximações e afastamentos da superfície terrestre, modelizando uma visualização telescópica, inclusive com a reprodução em desenho de seus dispositivos manuais de ajuste. No segundo, as linguagens do desenho, da história em quadrinhos, da perspectiva, do enquadramento cinematográfico, entre outras, modelizam um mundo paralelo virtual, no qual é possível simular praticamente todas as atividades de uma vida real: trabalho; lazer; namoro; esportes; conversas etc.

De um modo menos evidente, mas não menos importante, a web modeliza também outros tipos de espaço, notadamente, como iremos propor, o espaço público. Aqui podemos observar em maior intensidade a linguagem da programação visual como o principal sistema modelizante, diferentemente dos exemplos anteriores nos quais há uma presença mais marcante da fotografia ou do desenho como modelizadores de uma realidade virtual propriamente dita. Nesse caso, a distribuição espacial na interface, combinando diferentes linguagens, como a língua natural; a fotografia; a ilustração; as cores; os ícones, modeliza um ambiente de 
relacionamento social. Cabe destacar nesta programação visual, especialmente a modelização feita pela geometria, divisão do espaço da interface em quadrados e retângulos, e das cores, diferentes cores e tonalidades para definir as fronteiras entre diversos espaços. Dois exemplos dessa modelização do espaço público são as conhecidas redes sociais como o Orkut(7) ou o MySpace(8).

\subsection{O papel da eletricidade}

Por último, iremos trazer algumas pontuações de caráter especulativo na tentativa de introduzir uma exploração da dimensão da eletricidade presente nesta semiose. Para isso, lançaremos mão de algumas das idéias do pensador canadense Derrick de Kerckove. Para ele:

"Potencialmente, todas as tecnologias eletrônicas são interativas, estabelecem constantes e íntimas trocas de energia e de dados entre os nossos corpos e mentes e o ambiente global”. (DE KERCKHOVE, 1997, p. 248)

Para este autor a interatividade eletrônica é acima de tudo tato, na medida em que envolve muito mais do que um ponto de vista em perspectiva, mas verdadeiramente um ponto de existência, que percebe a realidade de modo proprioceptivo através de próteses tecnológicas de seus sentidos (visão, audição, tato e, em alguns casos, até mesmo olfato), como numa espécie de imersão no ambiente digital, que o integra a uma escala mundial de relações humanas e maquínicas.

Nesse ambiente, afirma Kerckhove, surge um novo tipo de espaço, que interliga de modo original o espaço público e o espaço privado. A eletricidade externaliza a mente dos indivíduos nas telas dos computadores, configurando uma mente coletiva e pública. O hipertexto promove, por sua vez, uma cognição compartilhada na medida em que permite o acesso a uma memória de todos. A velocidade tecnológica possibilita conexões cada vez mais amplas e mais rápidas, multiplicando geometricamente o potencial cognitivo.

A eletricidade, portanto, impulsiona a mente humana para uma outra dimensão perceptiva e cognitiva, que diz respeito não só à velocidade, mas notadamente à abrangência das interações, favorecendo sobremaneira as estratégias colaborativas (Cf. KERCKHOVE, 2003). Desta forma, promove a constituição de um espaço público estendido e coletivo que abre novos potenciais à mente humana, apontando para a constituição de uma inteligência coletiva.

O tema é bastante amplo e mereceria ser abordado em profundidade. Deixaremos, no entanto, essas idéias como inspiradoras para uma possível reflexão futura. De toda maneira, acreditamos que, mesmo colocadas de forma breve, servem para enriquecer a exploração sobre o tema deste trabalho: o contexto da semiose na cibercultura.

Para seguir nossa reflexão sobre a modelização do espaço público na web, iremos agora entrar diretamente na análise do website Overmundo, objeto privilegiado desta pesquisa, no qual queremos pontuar especialmente a existência de uma modelização da autoria oral em meio digital efetuada através da ação colaborativa no ambiente do espaço-informação.

\section{Análise do website Overmundo}

O Overmundo é um website que traz notícias sobre cultura brasileira produzidas pelo próprio público participante. Lançado em março de 2006, possui uma interface aberta e colaborativa, isto é, qualquer pessoa, desde que se registre no site, pode enviar matérias para uma das suas áreas editoriais: overblog; banco de cultura, guia e agenda. Seu processo editorial é feito de forma coletiva e descentralizada. As matérias enviadas entram primeiramente na fila de edição, por 48 horas, na qual podem receber comentários dos demais participantes. A seguir, vão para a fila de votação, por outras 48 horas, onde receberão os votos para sua 
publicação. Ao final deste período, se conseguirem atingir o número mínimo de votos, são publicadas na página de abertura, ganhando a maior visibilidade. As demais, são publicadas apenas no perfil de seu autor, ficando portanto pouco visíveis (9).

\section{Firefox}

- Favoritos Ferramentas Ajugda

6 [0. hetp://iwww.overmundo.com.br

$\rightarrow \mathrm{G} \cdot$

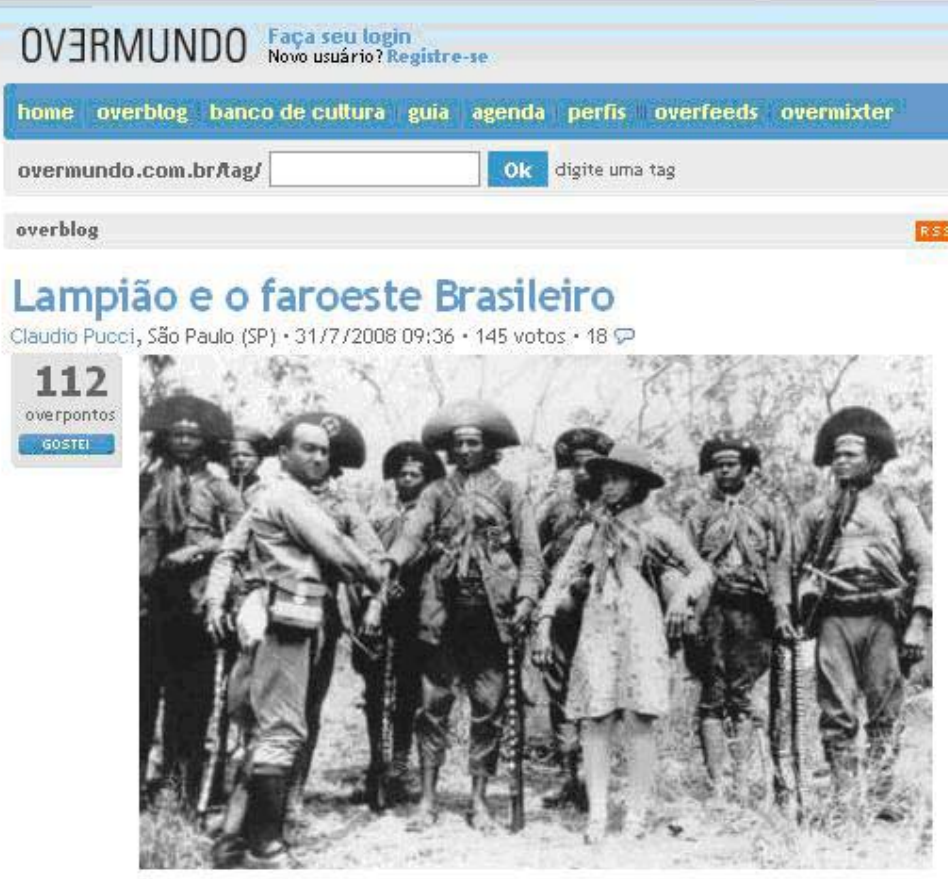

Não vi no UOL, nem no Terra, nem na Época, mas hoje é o aniversário de 70 anos da morte de Virgulino Ferreira, o Lampião. Figura controversa e mítica, Lampiäo mistura todas as qualidades e defeitos do bandido heróico, do considerado Robin Hood brasileiro pela revista Time de 1931, com uma pitada de lenda de faroeste americano. Muitos o acham um herói, outros um socialista, mas parece... $\geq$ mais

\section{A follha que sobrou do caderno (e da follha d} ponto)

Luana Wernik, Brasilia (DF) $\cdot 30 / 7 / 200823: 14 \cdot 94$ votos $\cdot 60$

46 Lembro perfeitamente de uma discussão que tive com um ex-chefe, onde 46 em resposta ao meu chamado para participação em listas de discussão ele overpontos respondeu: "Designers pensando coletivamente? Em que mundo você vive?". O que mais me assustou porém, foi o fato disso não ter partido de um publicitário ou marketeiro, e sim de um designer de produto. Há tempos formado, só quer saber da sua... s mais

\section{que o Senado tem de melhor}

Fabiana Mesquita, Rio Branco $(\mathrm{AC}) \cdot 30 / 7 / 2008$ 12:24 $\cdot 111$ votos $\cdot 40$

33 "Quando a velhice chegar, aceita-a, ama-a. Ela é abundante em prazeres

33 se souberes amá-la. os anos que văo gradualmente declinando estão entre

overpontos os mais doces da vida de um homem. Mesmo quando tenhas alcançado o

limite extremo dos anos, estes ainda reservam prazeres."

A frase de Séneca foi a última a preencher as impressões que absorvi

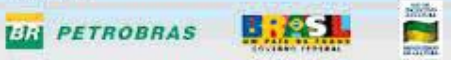

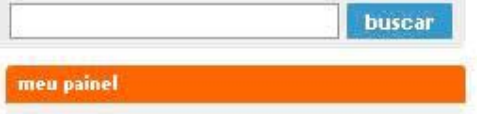

- publicar colaboração

$\Rightarrow$ filas de edição

- filas de votação

colaboraçōes por estado

veja colaborações referentes a um estado

Selecione... $\vee$

colaborações por assunto

Selecione... $\quad \checkmark$

tags randômicas

adeus globo tauro-gueluta roda-de-samba ceu janela

eduardo-ferreira

cadeiaprodutivadamusica

\section{nuvem de tags}

observatório

iSummit 2008

Começou ontem na cidade de Sapporo, no Japão, o isummit 2008. O evento é dedicado a discussões sobre cultura livre, e se baseia principalmente nas

O isummit é uma iniciativa do iCommons,

grupo que compartilha dos mesmos princípios

de cultura e educaça livre do nosso site. Năo ajudou, por... > leia

fóruns

Observatório - tudo sobre o Overmundo

Ajuda $\cdot$ tire suas dúvidas aqui

Código $\cdot$ sobre o sistema do site

Conversas $\cdot$ sobre culturas de todo o Brasil

Classificados $\cdot$ produtos e servicos culturais

Figura 1 - Interface da página de abertura do Overmundo

\subsection{Autoria colaborativa e espaço público no Overmundo}

No tipo de modelização do espaço público observado no website Overmundo constatamos que existem diferentes camadas de linguagens que garantem sua estruturalidade: num primeiro plano, temos o hipertexto 
que organiza e conecta as diferentes telas de informação através de elos de ligação semântica; em um segundo nível, observamos a presença da linguagem da programação visual definindo as fronteiras entre os diferentes espaços da interface através da combinação de elementos variados. Em uma terceira camada podemos considerar esses elementos - fotos; cores; língua natural; filmes; ilustrações etc. Assim, podemos visualizar a tessitura de múltiplos signos e linguagens em interação dinâmica na interface digital. Na Figura 1, podemos observar como diferentes linguagens se estruturam e dialogam entre si na interface da página de abertura do site.

O que propomos em nossa reflexão é a existência de uma modelização da autoria colaborativa nessa interface, estruturada em dois ambientes inter-relacionados, a saber, o da identidade e o da interação, que iremos analisar, respectivamente, como lugar de fala e espaço público no meio digital. Na interação entre esses dois elementos, verificamos a modelização de um diálogo, a qual compreendemos como um processo autoral.

O espaço público é modelizado na própria interface do website, com lugares definidos para o discurso e o diálogo - os espaços nos quais se inserem as colaborações (textos enviados pelos participantes) e os espaços nos quais se inserem os comentários (qualquer tipo de análise, elogio ou crítica ao texto enviado). Sendo assim, podemos pensar numa modelização da esfera pública, com a existência de um diálogo que se dá através da exposição de um discurso e de um meta-discurso, que seriam modelizadores de falas em um contexto da oralidade. Este espaço público modelizado é encontrado no website nas filas de edição, filas de votação e em todas as colaborações, pois em todos há sempre o discurso e, em potencial, o diálogo.

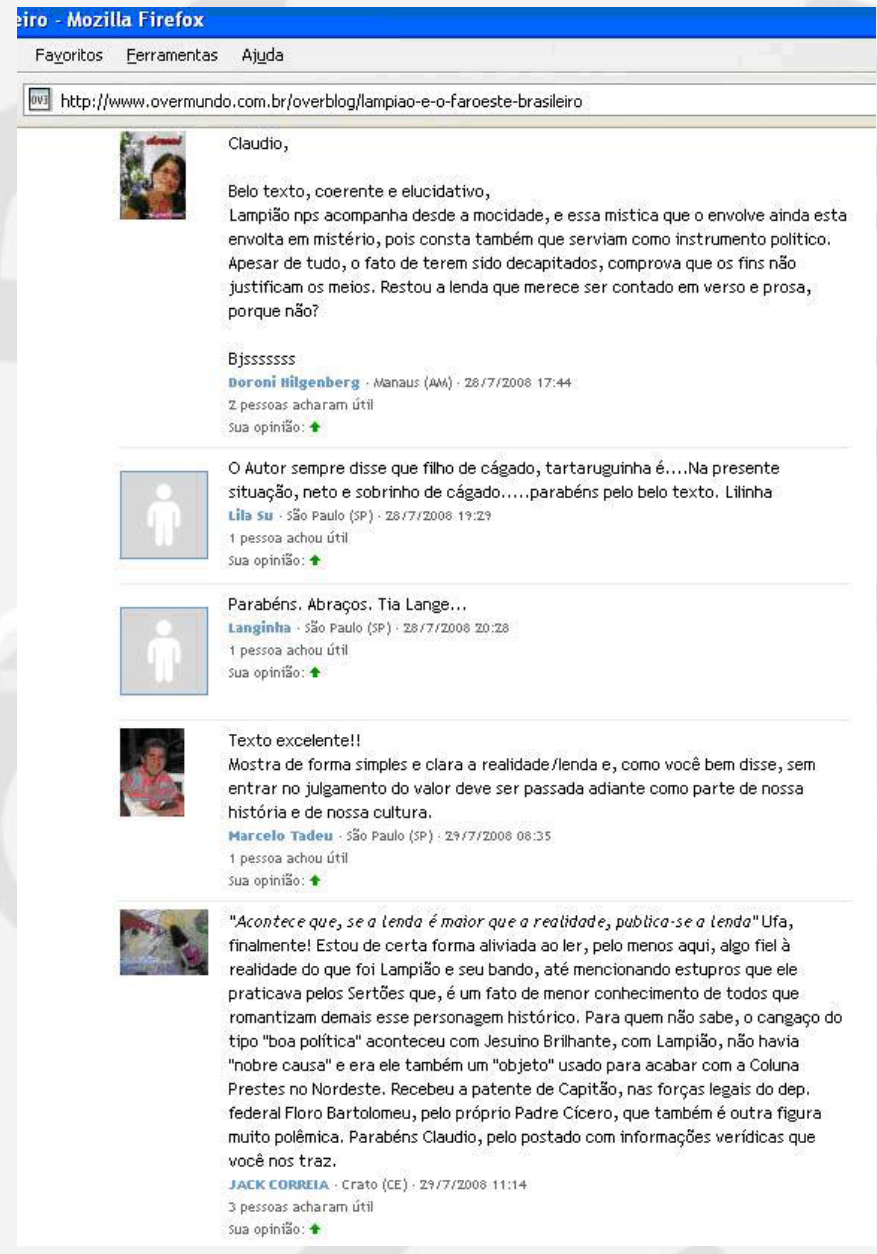

Figura 2 - Interface do espaço de comentários no Overmundo 
Na Figura 2 podemos observar a interface do espaço de comentários às colaborações no Overmundo. Dois pontos merecem ser ressaltados: as modelizações do diálogo e da identidade. Em um primeiro plano, o hipertexto estrutura as informações, conectando os diferentes espaços do website, inclusive o de comentários com cada uma das identidades ali representadas (10). Registramos a existência da modelização de um diálogo oral nesse ambiente, através das falas modelizadas em língua natural escrita e das representações das identidades, modelizadas ora em fotografia, ora em ilustração, cada uma delas rica em significações (uma foto pessoal; uma fotografia; um ícone padrão de perfil impessoal).

Nessa modelização autoral observamos ainda a existência de signos de interação entre os participantes. O mais importante deles é o perfil, que modeliza a identidade de cada um através de uma representação na interface digital, composta de signos discretos e não-discretos. O signo não discreto é uma foto ou ilustração que representa o participante em todos os comentários que faz no website. Como signo discreto há o nome ou pseudônimo, que acompanha todas as colaborações e comentários, e diversas informações que constam numa área específica do website chamada perfil: uma definição pessoal; suas colaborações ao projeto; seus autores favoritos dentro do Overmundo; as tags (palavras-chave) mais usadas (pode haver também fotos ou ilustrações nessa área). Deste modo o perfil modeliza uma identidade pessoal para cada participante: uma aparência que inclui um rosto (ou a representação de um rosto), uma definição de si, preferências etc. Este perfil é um elemento relacional no contexto da interação do espaço público modelizado. A Figura 3 traz um exemplo de perfil de participante ou colaborador do Overmundo.

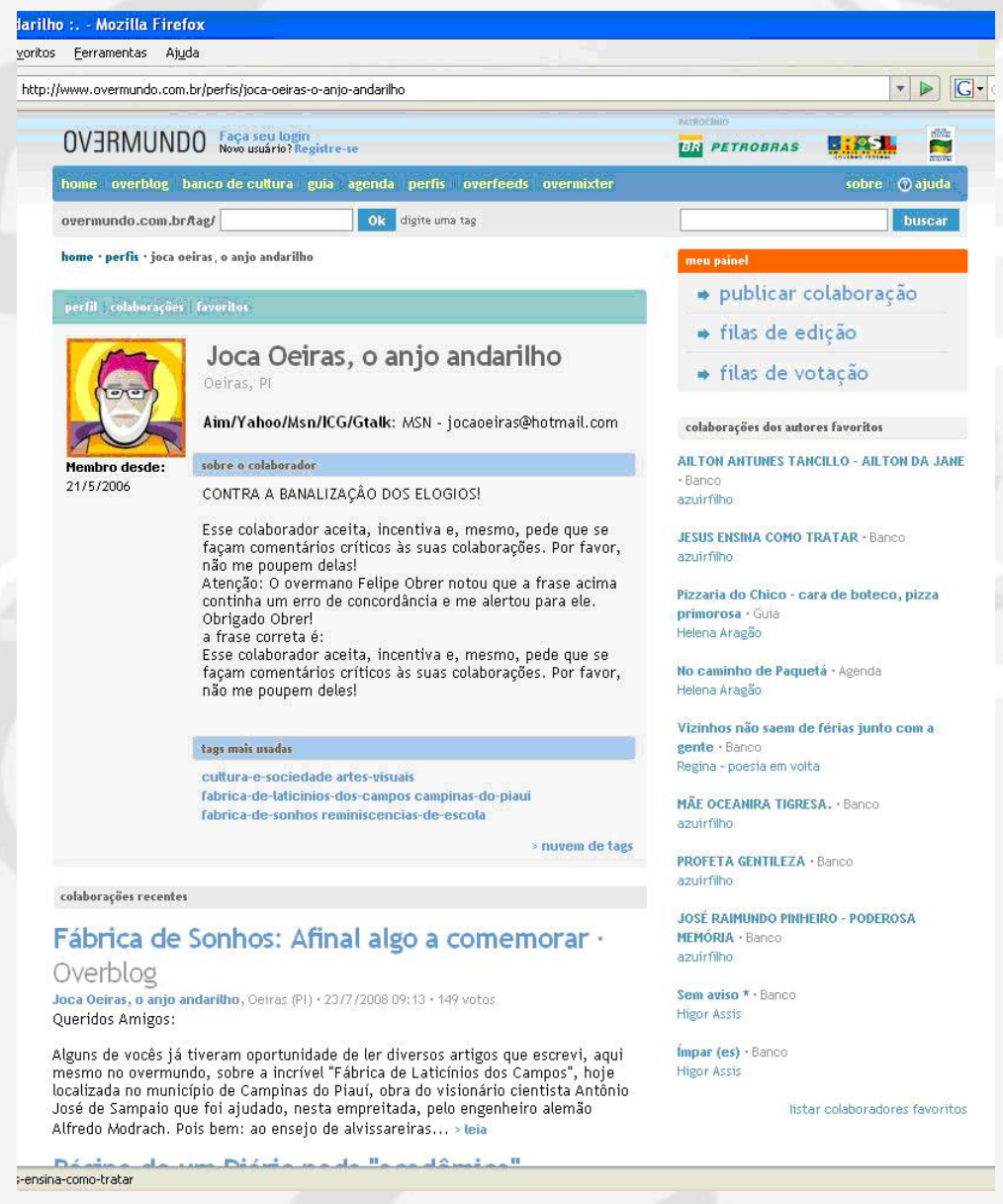

Figura 3 - Página do perfil de um dos participantes do Overmundo 
Por último, apontamos para a modelização de uma ação coletiva colaborativa: cada um dos discursos publicados pode ganhar comentários do público participante, e ser modificado, sofrer acréscimos ou cortes, de acordo com as sugestões, elogios ou críticas que receber. Deste modo há sempre a potencialidade, nem sempre efetivada, da construção de um texto - composto de conteúdo em língua natural, imagens e links - que seja o resultado de uma interação de falas diversas. Esta ação coletiva se estrutura através de um diálogo modelizado dentro da estrutura do espaço público digital já descrito anteriormente: os falantes modelizados em fotos ou imagens de seus perfis, por sua vez modelizam suas falas na língua natural escrita, e vão ali construindo um diálogo com direito a réplicas e tréplicas indefinidamente. A ação colaborativa, parte integrante da semiose, se apresenta, portanto, como esse diálogo no qual o novo vai sendo criado a partir da interação entre diferentes falantes, numa oralidade modelizada na interface do computador.

\section{Considerações finais}

No meio digital, constituído de várias camadas de linguagens modelizantes, observamos a emergência de um processo autoral colaborativo que segue as combinatórias de seu código: veloz; múltiplo; interativo; nãolinear, diverso, fragmentado, intertextual e transitório.

Inspirados em Kerckhove, podemos acrescentar ainda a eletricidade como um dos componentes desta semiose: a velocidade e a instantaneidade elétricas promovem o surgimento de uma mente coletiva, feita de memória compartilhada estruturada em múltiplos elos de ligação semântica.

O objeto apresentado aqui, o website Overmundo, é exemplar ao demonstrar a capacidade criativa e o potencial dessa autoria colaborativa feita da multiplicidade das interações. Procuramos descrever neste trabalho como se dá o processo dialógico e interativo entre diferentes sistemas de linguagens modelizados no ambiente eletrônico das redes de comunicação, que por sua vez modelizam complexas relações interpessoais e coletivas no que chamamos de espaço público virtual.

Este ensaio pretende ser um primeiro olhar, um tanto desbravador, na busca pela interpretação da cibercultura através dos instrumentos teóricos e metodológicos da Semiótica da Cultura. Esperamos que possa trazer alguma contribuição, senão suscitar algumas questões, para as pesquisas desse campo de estudos.

\section{Bibliografia:}

DE KERCKHOVE, Derrick. A pele da cultura. Lisboa: Relógio D’Água, 1997.

Texto, contexto, hipertexto: três condições da linguagem, três condições da mente.

In: Revista Famecos, Porto Alegre, n. 22, p.7-12, dez. 2003.

JOHNSON, S. Cultura da Interface, Como o Computador Transforma Nossa Maneira de Criar e Comunicar. Rio de Janeiro: Jorge Zahar Editor Ltda, 1997.

LEMOS, André. Cibercultura - tecnologia e vida social na cultura contemporânea. Porto Alegre: Sulina, 2002.

LOTMAN, Iuri. Introdução; Arte como linguagem A estrutura do texto artístico (trad. M.C.V. Raposo e A. Raposo). Lisboa: Estampa, 1978.

MACHADO, Irene. Impact or explosion? Technological culture and the ballistic metaphor. Sign Systems 
Studies no 34.1. Tartu: Tartu University Press, 2006.

PRIMO, Alex; RECUERO, Raquel. Hipertexto cooperativo: uma análise da escrita coletiva a partir dos Blogs e da Wikipédia. In: Revista Famecos, Porto Alegre, n. 22, p.54-65, dez. 2003.

\section{Notas:}

(1) Para um maior aprofundamento neste tema conferir PRIMO, Alex; RECUERO, Raquel. Hipertexto cooperativo: uma análise da escrita coletiva a partir dos Blogs e da Wikipédia. In: REVISTA FAMECOS, Porto Alegre, n. 22, p.54-65, dez. 2003.

(2) Espaço-informação é o conceito proposto por Steven Johnson para pensar o ambiente da interface das redes de comunicação. Cf. JOHNSON, S. Cultura da Interface, Como o Computador Transforma Nossa Maneira de Criar e Comunicar. Rio de Janeiro: Jorge Zahar Editor Ltda, 1997.

(3) Endereço eletrônico em: http://www.overmundo.com.br.

(4) Neste artigo não teremos espaço para desenvolver o conceito de semiosfera. Para um aprofundamento no tema, conferir LOTMAN, I. "Acerca de la semiosfera". La semiosfera I. Semiótica de la cultura y del texto (Desidério Navarro, org.). Madrid: Cátedra, 1998.

(5) Endereço eletrônico em: http://earth.google.com/intl/pt/.

(6) Endereço eletrônico em: http://secondlife.com/.

(7) Endereço eletrônico em: http://www.orkut.com.

(8) Endereço eletrônico em: http://www.myspace.com/.

(9) Informações disponíveis em: http://www.overmundo.com.br/estaticas/ajuda.php. Acesso em: 20 jul 2008.

(10) Cada uma das identidades possuem links para a página do perfil correspondente.

\section{Mini Currículo :}

Mestre em Comunicação e Cultura pela Escola de Comunicação da UFRJ. Doutoranda do Programa de PósGraduação em Ciências da Comunicação da Escola de Comunicações e Artes da USP. Pesquisadora do Colabor-USP - Núcleo de Pesquisa em Linguagens Digitais. 